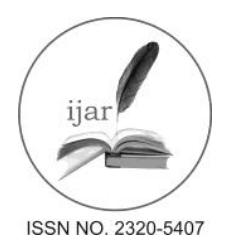

Journal homepage:http://www.journalijar.com
Journal DOI:10.21474/IJAR01

RESEARCH ARTICLE
INTERNATIONAL JOURNAL

OF ADVANCED RESEARCH

\title{
SYNTHESIS OF ADAPTIVE ANTENNA ARRAYS WITH VARIABLE ELEMENT SPACING USING LMS
} ALGORITHM.

\author{
*Somnath Patra ${ }^{1}$, Subhrajit Paul ${ }^{2}$, Sumit Ghosh ${ }^{2}$, Souvik Kumar Guin ${ }^{2}$, Rikta Pramanik ${ }^{2}$ Biswajit Mahanty $^{1}$. \\ 1. Asst. Prof. ECE department SMIT (Techno India Group), Hooghly, India. \\ 2. 4th year ECE department, SMIT (Techno India Group), Hooghly, India.
}

\section{Manuscript Info Abstract}

Manuscript History:

Received: 25 April 2016

Final Accepted: 19 May 2016

Published Online: June 2016

Key words:

Beamformed adaptive system, MSE, LMS

*Corresponding Author
Adaptive/Smart antenna arrays allowed an antenna to steer the beam to any direction of interest (DOI) while simultaneously nulling interfering signals. In this paper an approach based on Least Mean Square (LMS) is incorporated to minimize the SLL and MSE of adaptive antenna arrays by varying the interelement spacing in between $0.5 \lambda$ to $1.0 \lambda$ with DAOA 10 deg. and IAOA 70 deg. LMS is a one of the most efficient gradient based approach of steepest decent that makes correction to the weight vector to minimize the MSE. After comparing with previous results of uniformly spaced elements with our proposed approach, superiority of our proposed approach is established.

Somnath Patra.

Copy Right, IJAR, 2016,. All rights reserved.

\section{Introduction:-}

Adaptive/Smart antenna arrays have numerous benefits in wireless applications, sensors as well as in radars. Adaptive antenna arrays usually comprise in both switch beam and beam formed adaptive system. A switched beam system operates on several fixed beam patterns. Beamformed adaptive systems have the capability to allow antenna, steer the beam to any direction of interest while continuously nulling interfering signals. Adaptive antennas provide a narrow beam towards the higher system capacity with the users of interest, while nulling the not interested users. This provides, low power level with high SNR and permits greater frequency to reuse within the same cell, this concept is called as SDMA. Adaptive antennas can be utilized to increase direction-finding (DF) techniques by more correctly finding the angle-of-arrival (AOA) [1-3]. A large number of arrays with spectral estimation techniques can be incorporated, which can be able to isolate the AOA with an angular precision that surpass the array resolution. Adaptive antennasdirection finding capabilities increase the geo-location services by enabling a wireless system to better determine the location of a particular mobile user. Additionally, adaptive antennas can direct the main beam towards the signals of interest even without any reference signal or training sequence, this capability is called blind adaptive beamforming. Adaptive/Smart antennas also called as digital beamformed (DBF) arrays [4] or adaptive arrays (depending upon the adaptive algorithms utilized). Adaptive antennas power patterns are controlled via algorithms depends upon some unique criteria. These criteria could be minimizing the mean square error (MSE), minimizing the variance, steering towards a signal of interest (SOI), maximizing the signal-to-noise interference ratio (SIR), nulling the interfering signals, or tracking a moving emitter to name a few. When the algorithms used are adaptive in nature, this process is referred to as adaptive beamforming [5]. Recent works are being exerted to modify radar system to include adaptive beamforming techniques for commercial purpose. In this paper, an adaptive/smart antenna array system is optimized using a distinct adaptive algorithm, Least Mean Square (LMS), after comparison, the optimized results obtained from this algorithm with previously obtained results, superiority of the proposed approach will be disclosed. 


\section{Problem Formulation:-}

An adaptive/smart antenna array is considered here. Adaptive beamforming [6], [7] is more effective and useful beamforming solution because of the digital beamformer, simply consists of an algorithm which can dynamically optimize the array pattern according to the change occurs in the electromagnetic environment. In conventional static array processing systems are subject to downcast by various causes. The array SNR can be severely degraded by the presence of unwanted interfering signals. Beamforming [8] is usually accomplished by phasing the feed to each element of array so that signals transmitted or received from all the elements in phase in a certain direction. The array factor for $N$ elements linear array with variable element spacing is given by,

$$
F(\theta)=\sum_{n=0}^{N-1} A_{n} e^{\left(j n\left(\frac{2 \pi d}{\lambda} \cos (\theta)+\beta_{i}\right)\right)}
$$

where, inter-element phase shift,

$$
\begin{aligned}
& \left(\beta_{i}\right)=-\frac{2 \pi d_{x}}{\lambda_{0}} \cos \left(\theta_{0}\right) \\
& \lambda_{0}=\text { desired wavelength. } \\
& \theta_{0}=\text { desired beam direction. } \\
& d_{x}=\text { variable inter-element spacing. }(0.5 \lambda-1.0 \lambda) .
\end{aligned}
$$

The array output $y(t)$ is the weighted sum of the received signals. $S_{n}(t)$ at the array elements and the noise $x(t)$ at the receiver which are connected to each array element. The weights iteratively computed based on the output array $y(t)$, the reference signal $r(t)$ that approximates the desired signal and previous weights. The reference signal is approximated to the desired signal using a spreading sequence.

The array output is given by,

$\mathrm{y}(\mathrm{t})=\omega_{n}{ }^{\mathrm{H}} * \mathrm{x}(\mathrm{t})(2)$

Where, $\omega^{H}$ denoted as the transpose of complex conjugate for the weight vector $\omega$.

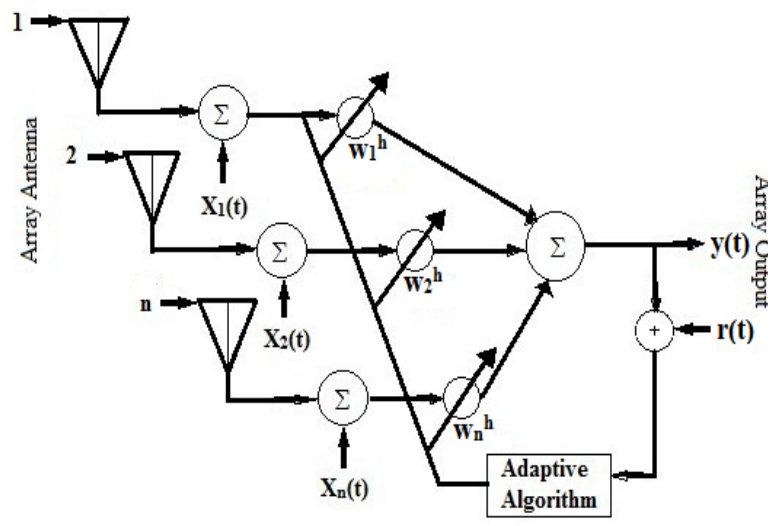

Fig.1:- An adaptive antenna array arrangement.

In order to compute the optimum weights of the steering or array response vector from the sampled data of the array output has to be known. The array response vector is the function of incident angle as well as the frequency. The baseband received signal at the $N^{\text {th }}$ antenna is sum of phase shifted and attenuated version of the desired signal $S_{i}(t)$.

Where, $\omega^{H}$ denoted as the transpose of complex conjugate for the weight vector $\omega$. In order to compute the optimum weights of the steering or array response vector from the sampled data of the array output has to be known. The array response vector is the function of incident angle as well as thefrequency. The baseband received signal at the $N^{\text {th }}$ antenna is sum of phase shifted and attenuated version of the desired signal $S_{i}(t)$. 


$$
X(t)=\sum_{i=1}^{N} a_{i}\left(\theta_{i}\right) S_{i}(t) e^{-j 2 \pi f_{c} \tau_{i} \theta_{i}}
$$

The $S_{i}(t)$ is combination of both desired signal and the interfering signal. The beam former response can be expressed in vector form as,

$$
r(\theta, \omega)=\omega_{n}{ }^{H} * a(\theta, \omega)_{(4)}
$$

This equation is includes the possible dependency of a $\theta$ on $\omega$ as well. The proposed adaptive array configuration which is consist of the antenna array elements terminated in an adaptive processor which is designed to specifically maximize certain criteria as the emitters move or change, the adaptive array updates and compensates iteratively in order to track the changing environment.

\section{Brief overview of LMS algorithm:-}

The Least Mean Square (LMS) algorithm is a gradient-based method of steepest decent. Monzingo [9-12] gives an excellent fundamental treatment of this approach. Gradient based algorithms assume an established quadratic performance surface. LMS uses the estimates of the gradient vector from the available data and incorporates an iterative procedure for successive corrections to the weight vector in the direction of the negative of the gradient vector which eventually leads to the minimum MSE compared to other algorithms. LMS algorithm is relatively very easy to implement .We can establish the cost function by again finding the MSE. The error, as indicated is,

$$
\varepsilon(k)=d(k)-\bar{w}^{H}(k) \bar{x}(k)_{(5)}
$$

The squared error is given as

$$
|\varepsilon(k)|=\left|d(k)-\bar{w}^{H}(k) \bar{x}(k)\right|^{2}
$$

The cost function is given as

$$
j(\bar{w})=d-2 \bar{w}^{H} \bar{r}+\bar{w} \bar{R}_{x x}(\bar{w})
$$

The solution for the weights is the optimum Wiener solution as given by

$$
\overline{w_{\text {opt }}}=\bar{R}_{x x}^{-1} \bar{r}
$$

Where $\bar{R}_{x x}^{-1}=$ Array correlation matrix.

$\bar{r}=$ the signal correlation vector.

In general, we do not know the signal statics and thus must resort to estimating the array correlation matrix $\left(\overline{R_{x x}}\right)$ and the signal correlation vector $(\bar{r})$ over a range of snapshots of for each instant in time.

The instantaneous estimates of these values are given as

$$
\begin{aligned}
& R_{x x}(k) \approx \bar{x}(k) \overline{x^{H}}(k) \& \\
& \hat{r}(k) \approx d_{n}^{*}(k) \bar{x}(\mathrm{k})
\end{aligned}
$$

If we substitute the instantaneous correlation approximation, we have the LMS solutions.

$$
\bar{w}(k+1)=\bar{w}(k)-\mu\left[R_{x x} \bar{w}-\hat{r}\right]=\bar{w}(k)+\mu e^{*}(k) \bar{x}(k)(8)
$$

Where the error function is given by as

$\mathrm{e}(\mathrm{k})=d_{n}(k)-w * h(k) \bar{x}(\mathrm{k})$

Where, $e(k)=$ Error signal,

$d_{n}(k)=$ Reference signal,

$$
\bar{x}(k)=\overline{x_{s}}(k)+\bar{x}_{i}(k)+\bar{n}(k)
$$


$\overline{x_{s}}(\mathrm{k})=$ desired signal vector.

$\overline{x_{i}}(\mathrm{k})=$ interfering signal vector .

$\bar{n}(\mathrm{k})=$ zero mean Gaussian noise for each channel.

\section{Results and Discussion:-}

Let consider, a linear antenna array with 10 elements andvarying inter-element spacing (ranges in between $0.5 \lambda$ to $1.0 \lambda$ ) with desired $\mathrm{AOA}=10 \mathrm{deg}$. and interferer $\mathrm{AOA}=70 \mathrm{deg}$. is considered, where total number of data samples taken as 100. All the elements are uniformly excited with fixed amplitude values. Adaptive/Smart antenna array is optimized using LMS algorithm.

Table I:- Optimization parameters utilize to synthesize adaptive antenna arrays using LMS algorithm.

\begin{tabular}{|c|c|c|c|c|c|}
\hline $\begin{array}{c}\text { No. of } \\
\text { Observations }\end{array}$ & $\begin{array}{c}\text { Element number } \\
(\mathbf{N})\end{array}$ & $\begin{array}{c}\text { Element } \\
\text { spacing (d } \mathbf{x})\end{array}$ & $\begin{array}{c}\text { Desired AOA } \\
\text { (Deg.) }\end{array}$ & $\begin{array}{c}\text { Interferer AOA } \\
\text { (Deg.) }\end{array}$ & $\begin{array}{c}\text { No. of iteration } \\
\text { to minimize MSE }\end{array}$ \\
\hline 1 & 10 & 0.65 & 10 & 70 & 43 \\
\hline 2 & 10 & 0.80 & 10 & 70 & 45 \\
\hline 3 & 10 & 0.90 & 10 & 70 & 48 \\
\hline 4 & 10 & 0.50 & 10 & 70 & 51 \\
\hline
\end{tabular}

In Table. I various optimization parameters are shown, it is clearly observed that all the parameters are static in nature except the element spacing and MSE. In our proposed approach our main aim to minimize the MSE by varying the inter-element spacing.

Optimized power patterns using LMS is shown in fig.2, fig.3, fig.4 and fig.5. which represents the minimized MSE with respect to iteration number. As in [13] we have taken the number of element $(\mathrm{N})=10, D A O A=10 \mathrm{deg}, I A O A=70$ deg, but varying the inter-element spacing from $0.5 \lambda$ to $1.0 \lambda$, then it isclearly observed that by thevariation of the element spacing superior change in MSE is occurred as shown in fig.2. where the MSE is minimized after $42^{\text {th }}$ iterations.

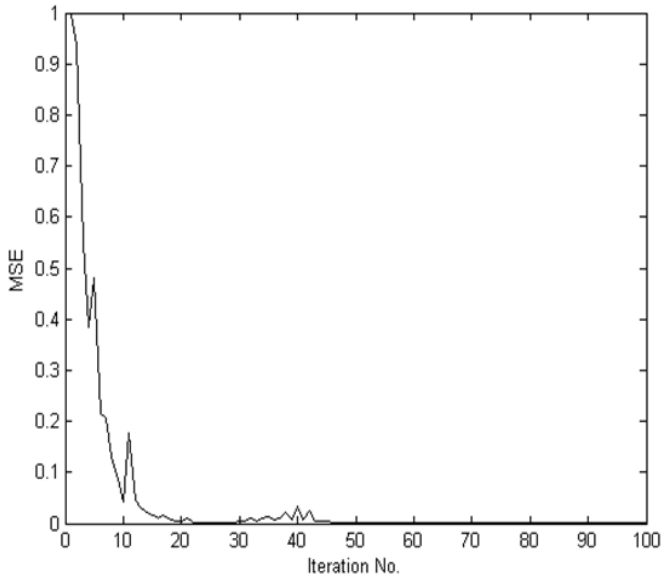

Fig.2:- MSE obtained using LMS algorithm for $\mathrm{N}=10$, $\mathrm{DAOA}=10$ deg., $\mathrm{IAOA}=70$ deg., with $0.65 \lambda$ spacing.

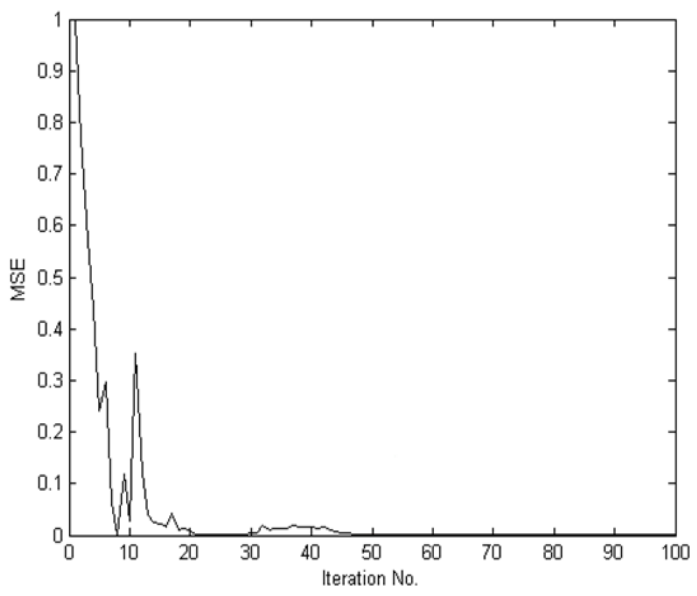

Fig.3:- MSE obtained using LMS algorithm for $\mathrm{N}=10$, $\mathrm{DAOA}=10$ deg., $\mathrm{IAOA}=70$ deg., with $0.80 \lambda$ spacing.

In fig. 3 and fig. 4 the MSE is minimized only after $44^{\text {th }}$ and $47^{\text {th }}$ iterations which is less time consuming than the uniformly spaced adaptive antenna arrays as in [11][13], the MSE is minimized after $50^{\text {th }}$ iterations shown in fig. 5. After comparing these optimized results the superiority of our proposed approach is established. 


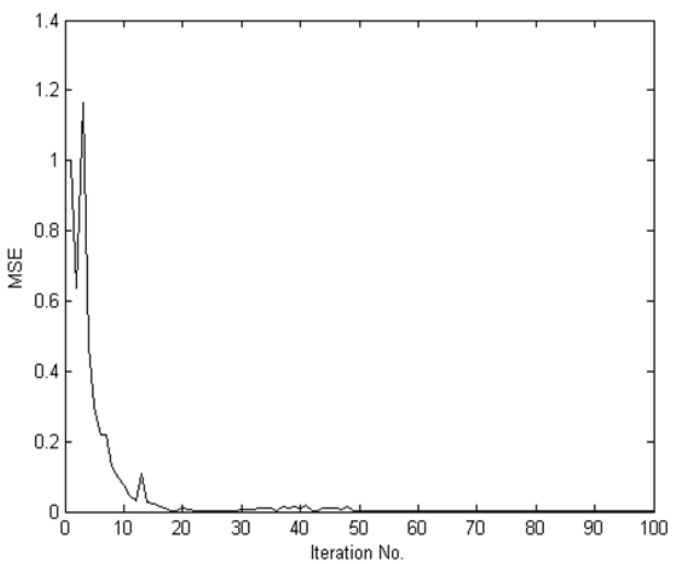

Fig.4:- MSE obtained using LMS algorithm for $\mathrm{N}=10$, $\mathrm{DAOA}=10 \mathrm{deg}$., IAOA=70 deg., with $0.90 \lambda$ spacing.

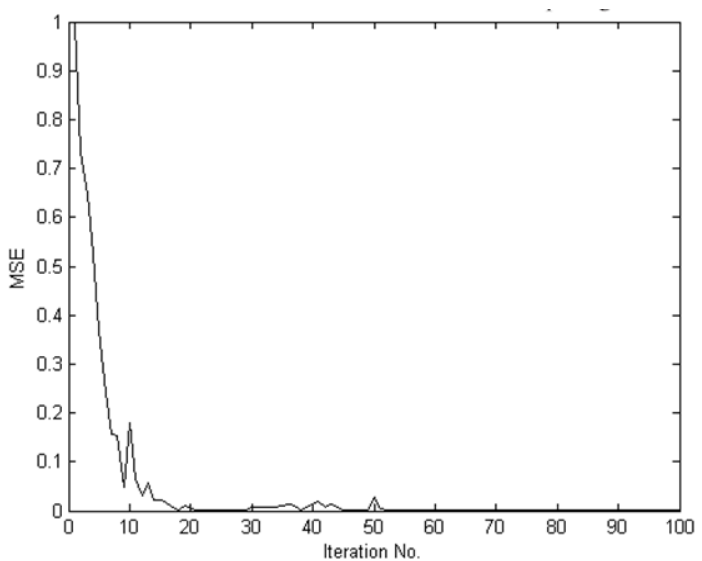

Fig.5:- MSE obtained using LMS algorithm for $\mathrm{N}=10$, $\mathrm{DAOA}=10 \mathrm{deg}$., IAOA=70 deg., with $0.50 \lambda$ spacing.

\section{Conclusion:-}

In this article an adaptive antenna array is optimized using LMS algorithm. As LMS algorithm have some disadvantages like it must go through many iterations before satisfactory convergence is achieved. One of the possible approaches to circumventing the relatively slow convergence of the LMS scheme is by use of variable inter-element spacing. In case of uniform element spacing in adaptive arrays, when LMS is applied the MSE is minimized after $50^{\text {th }}$ iterations but in case of variably spaced elements, the MSE is minimized only after $42^{\text {th }}$ iterations. That signifies the superiority of the proposed approach over conventional approaches. This algorithm has been simulated using MATLAB 8.1 (R2013a) version software in Windows 7 (32 bit) environment.

\section{References:-}

1. C. S. Rani, P. V. Subbaiah, K. C. Reddy (2008),LMS and RLS Algorithms for Smart antennas in a CDMA Mobile Communication environment, International Journal of the Computer, the Internet and Management, Vol.16, No.3, pp. 12-21.

2. B. Widrow and S. D Streams (2005), Adaptive Signal Processing, Person Education, New Delhi.

3. C. BALANIS (2011), Antenna Theory- Analysis and design. New York: Wiley.

4. F. B. Gross (2005), Smart Antenna for Wireless Communication. McGraw-Hill Companies.

5. J. R. Treichler (2004), Theory and Design of Adaptive Filters, Prentice-Hall of India.

6. J. C. Liberti, Jr. (1999),Smart Antennas for Wireless Communications: IS-95 and Third Generation CDMA Applications, Prentice Hall PTR.

7. L.Godara (1997), Application of antenna arrays to mobile communications, Part-II: Beamforming and directional of arrival considerations IEEE Proceedings, Vol. 85, No.8, pp. 1195-1245.

8. M. Yasin, P. Akhtar (2010), "Performance Analysis of LMS and NLMS Algorithms for a Smart Antenna System”, International Journal of Computer Applications, Vol.4,No.9,pp.25-32.

9. Oeting, J. (1983), Cellular Mobile Radio- An Emerging Technology, IEEE Communications Magazine, November, pp.10-15.

10. S.Patra,A.Pandey,N.Nandni,S.Kumar,S.Jha (2015), “Power pattern synthesis of smart antenna array using different adaptive algorithms", International Journal of Advanced Research, Vol.3,Issue 5,pp.1459-1466.

11. S.Patra, B.Mahanty (2014),"Synthesis of Smart Antenna Array Using Different Adaptive Algorithms", International Journal of Electronics and Communication Technology, Vol.5, Issue 3, July-Sept, pp.179-182.

12. S. Patra, N. Nandni, A.Pandey, S. Kumar (2014), "Study the Behavioral Change in Adaptive Beamforming of Smart Antenna Array Using LMS and RLS Algorithms", International Journal of Engineering Trends and Technology, V17 (7), pp. 310-314.

13. S. Singh, M. Kaur (2015), “LMS and NLMS Algorithm for Smart Antenna” IJARCSSE, vol.5, issue 4. 